\title{
Synthesis, Characterization and Application of Norm Encapsulated Zinc Phthalocyanine Nanoparticles as Photo Dynamic Therapeutic Agents
}

\author{
Tarun Mohan ${ }^{1,2 *}$, Hemant Kumar ${ }^{1}$, L. Krishna Bharat ${ }^{3}$ and Indrajit \\ $\operatorname{Roy}^{1 *}$ \\ ${ }^{1}$ Department of Chemistry, University of Delhi, Delhi, India \\ ${ }^{2}$ Government Degree College, Gairsain, Uttarakhand, India \\ ${ }^{3}$ Federal State Budgetary Scientific Institution "Federal Scientific Agroengineering \\ Center VIM" (FSAC VIM), Moscow, Russia \\ *Corresponding Author: Tarun Mohan Department of Chemistry, University of \\ Delhi, Delhi, India. E-mail: tarunmohandu@gmail.com and Indrajit Roy Department \\ of Chemistry, University of Delhi, Delhi, India. E-mail: indrajitroy11@gmail.com
}

\author{
Received: May 25, 2021 \\ Published: June 09, 2021 \\ (C) All rights are reserved by Tarun Mohan., \\ et al.
}

\begin{abstract}
Nanosized organically modified silica (NORM) particles are promising platforms for encapsulating/conjugating active agents for petitions in bioimaging, light activated therapies and drug delivery. Photodynamic therapy (PDT) is an encouraging and significant therapeutic technique in which light is used to activate photosensitizer molecules, that further reacts with molecular oxygen to produce highly reactive and cytotoxic singlet oxygen. Localized PDT can lead to selective killing of malicious cancer cells. Herein, we have synthesized zinc phthalocyanine (ZnPc) encapsulated NORM particles (ZnPc/NORM), in which ZnPc acts as the photosensitizer and NORM particles act as a carrier and also stabilizer of ZnPc. Further characterization of nanoparticles has been done by various techniques to study the morphology, structure and optical properties. ABMDMA dye was used to evaluate the photodynamic property of ZnPc/NORM particles. These nanoparticles have great potential for being a PDT agent as these nanoparticles significantly generate singlet oxygen on irradiation with laser light, along with some hyperthermia (rise in temperature). In vitro studies have been carried out using adenocarcinomic human alveolar basal epithelial (A549) cells to study the anticancer PDT efficacy of ZnPc/NORM.
\end{abstract}

Keywords: NORM; Zinc Phthalocyanine; Photodynamic Therapy; Hyperthermia; Cell Viability Assay

\section{Introduction}

In recent years chemotherapy, radiotherapy, surgery and few other methods were used for cancer treatment [1-3]. Although these techniques prove to be effective, one of the main issue encountered in treatment is the destruction of healthy cells along with cancerous cells. To overcome these complications photodynamic therapy (PDT) is actively used in treating several cancers in the clinic, along with dermatological disorders and microbial infections [4-6], etc. PDT is a non-invasive technique in which a photosensitizer drug is activated with light for the transmutation of molecular oxygen into the cytotoxic singlet oxygen $[7,8]$. Pure photosensitizer alone is harmless and have no impact on either abnormal or healthy tissues in the absence of light. PDT has many advantages over other therapies such as highly localized, cost effective, specific tumor treatment, and higher cure rate for some tumors, etc. [9-11].

Phthalocyanines belongs to the family of macrocycles which contains extensively delocalized 18 -匹 electrons system. Their structural planarity makes them insoluble in common organic solvents [12-14]. Owing to their excellent singlet oxygen photogeneration, phthalocyanines have been applied in PDT as efficient photosen- 
sitizers [15-19]. However, owing to their poor aqueous solubility, they have been formulated in several micro and nano-sized delivery systems for stable aqueous dispersion [20-25]. These delivery systems also ensure sustained release, avoid plasmatic fluctuation, reduce side effects, decrease the frequency of administration and enhance the drug uptake by targeted tissues, thereby improving the treatment potency $[26,28]$. For example, the hydrophobic zinc phthalocyanine was incorporated in a liposomal formulation for successful delivery [29,30]. Zinc phthalocyanine has powerful absorbance (red region) of spectrum of visible light, and a powerful emission in the range of $600-800 \mathrm{~nm}$ [31]. Light activated CP-NPs experience singlet $\left({ }^{1} \mathrm{O}_{2}\right)$ to triplet $\left({ }^{3} \mathrm{O}_{2}\right)$ intersystem crossing because of charge transfer and simultaneously nonradiative decay under fixed near infrared light irradiation wavelength. This phenomenon produce singlet oxygen quantum yield and elevated photothermal efficacy [32]. Keeping this in view, we have prepared a nanoparticle-based delivery system in which photosensitizer (ZnPc) was encapsulated inside the nanosized organically modified silica (NORM) particles. Silica-based nanoparticles are efficient carrier of biomolecules due to their high stability, durability, and easily modifiable surface chemistry. NORM particles have a large surface area, along with chemical, colloidal and thermal stability, which makes them suitable for their use as carrier agents [33-35]. NORM can easily penetrate cancer cells without causing any significant cytotoxicity in vitro [36-39]. After thorough characterization of the ZnPc-encapsulated NORM (ZnPc/NORM) particles, we have investigated their singlet oxygen photogeneration efficiency and light-activated toxicity in cancer cells in vitro.

\section{Experimental Procedure}

Materials

9,10-anthracenediyl-bis(methylene)dimalonic acid (ABMDMA), vinyltriethoxysilane (VTES), aminopropyl tetraethoxysilane (APTES), and n-butanol were purchased from Sigma-Aldrich Co. India. Dioctyl sulfosuccinate sodium salt (AOT), Dimethyl sulfoxide (DMSO), phthalonitrile, zinc chloride $\left(\mathrm{ZnCl}_{2}\right)$, lithium pentoxide $\left(\mathrm{Li}_{2} \mathrm{O}_{5}\right)$, acetone, methanol, ammonia, and concentrated $\mathrm{H}_{2} \mathrm{SO}_{4}$ were purchased Merck Darmstadt (Germany). Fetal Bovine Serum (FBS), antibiotics (streptomycin and penicillin), Dulbecco's Modified Eagle Medium (DMEM), and MTT reagent [3-4,5-dimethylthiazzol2-yl)-2,5-diphenyltetrazoliumbromide] were acquired from Genetix (India). A-549 cell lines were obtained from National Centre for
Cell Science (NCCS), Pune, India. Double distilled water was used during the experiment.

\section{Synthesis of $\mathrm{ZnPc}$}

A mixture of $\mathrm{ZnCl}_{2}(0.25 \mathrm{mmol})$ and phthalonitrile (1mmol) was refluxed under the purged nitrogen atmosphere in the solution of $\mathrm{Li}_{2} \mathrm{O}_{5}(10 \mathrm{ml})$ with constant stirring for 8 hours at $70^{\circ} \mathrm{C}$. The product was cooled to RT and after that methanol $(20 \mathrm{ml})$ was added [30]. The prepared zinc-phthalocyanine was separated and filtered to obtain a solid product. The solid product was washed with DMSO and acetonitrile for removing unreacted and side products. The synthesized ZnPc (PS) was further dried in an oven at ambient atmosphere for $24 \mathrm{~h}$ at $150^{\circ} \mathrm{C}$ and used for further characterization and applications.

\section{Synthesis of ZnPc/NORM}

A, oil-in-water microemulsion was ready by mixing $0.22 \mathrm{~g}$ of the surfactant (AOT) in $10 \mathrm{ml}$ of $\mathrm{H}_{2} \mathrm{O}$, along with $400 \mu \mathrm{L}$ of co-surfactant (n-butanol) [33]. To this $50 \mu \mathrm{l}$ of PS (1 mg/ml) in DMSO was added and the solution was vigorous stirring for 1 hour. Later, 100 $\mu \mathrm{L}$ of VTES was added and the resulting solution was vigorously stirred for 1 hour. Finally, $10 \mu \mathrm{l}$ of APTES was added to the solution and left overnight under stirring for nanoparticle formation. At the end of the synthetic process a bluish translucent solution was seen indicating the formation of nanoparticles. The surfactant, co-surfactant, and extra unreacted molecule present in the solution were eliminated by dialysis against double distilled water for nearly $48 \mathrm{~h}$, employing a cellulose dialysis membrane with a cut off size of 12-14 $\mathrm{kDa}$. At the edge of dialysis, the nanoparticles were sterile filtered utilizing a $0.45 \mu \mathrm{m}$ syringe filter and stored below at $4^{\circ} \mathrm{C}$ for future use.

\section{Characterization}

The ZnPc/NORM particles were characterized using different techniques to confirm the morphological, structural, and optical properties. The shape, size and morphology of the synthesized nanoparticles were confirmed by Scanning (SEM-JEOL JSM$6610 \mathrm{LV}$ ) electron microscope and transmission (TEM-TECNAI G2-30 U TWIN) electron microscope. The size range of the formed nanoparticle was confirmed by the dynamic light scattering technique (DLS-NANO-JS series Malvern Zetasizer). The structural characterization was done by x-ray diffractometer (XRD: Bruker D8 Discover), Fourier transform infrared spectrometer (FTIR: Per- 
kin Elmer RXI spectrometer), nuclear (NMR- ECX-400PNMR at 400 $\mathrm{MHz}$ and $100 \mathrm{MHz}$ ) magnetic resonance, and mass (MS: MALDI-TOF spectrometer: Agilent Technology-650) spectrometer. The optical spectra of the nanoparticles were observed by UV-Vis (SHIMADZU UV-1601, JAPAN) and fluorescence (Varian Palo Alto, CA) spectrophotometer.

\section{Photobleaching of ABMDMA dye}

In this experiment for singlet oxygen detection $\left({ }^{1} \mathrm{O}_{2}\right), 1 \mathrm{mg} / \mathrm{ml}$ of the stock solution of Na salt of ABMDMA $(16 \mu \mathrm{M})$ in $\mathrm{H}_{2} \mathrm{O}$ was prepared and sonicated with pure ZnPc or ZnPc/NORM $(100 \mu \mathrm{g} /$ $\mathrm{mL}$ ) nanoparticles. The final concentration of $\mathrm{ZnPc}$ was $8.0 \mu \mathrm{M}$. The prepared solutions were continuously stirred and irradiated in a quartz cuvette using a power tunable diode laser of $635 \mathrm{~nm}$ wavelength and power of $38 \mathrm{~mW} / \mathrm{cm}^{2}$ for different periods of 5 , 10 , and 15 mins. Finally, the optical density was measured for all the samples using the UV-Vis spectrophotometer. Reduction in the optical density of ABMDMA peaks (around $400 \mathrm{~nm}$ ) directly correlated with the ${ }^{1} \mathrm{O}_{2}$ production.

\section{In vitro studies}

The A-549 (Adenocarcinomic human alveolar basal epithelial) cells, cultured under optimum growth conditions, were used for in vitro studies. At a cell confluence of about 75 - 80\% (102 cells/ mL media), the cell cytotoxicity assay was performed. A sterile 24-well cell culture plate was taken in which four different concentrations of NPs $(20,50,100,150,200,250,300 \mu \mathrm{g} / \mathrm{ml})$ were added in separate wells, mixed gently, and incubated for $48 \mathrm{~h}$ in dark. Irradiation in desired wells was done just after the $2 \mathrm{~h}$ of incubation by a $635 \mathrm{~nm}$ wavelength red laser light (power density $40 \mathrm{~mW} / \mathrm{cm}^{2}$ ) and further incubated for $48 \mathrm{~h}$. After incubation, each well was thoroughly washed with sterile PBS and $100 \mu \mathrm{L}$ of MTT reagent (5 $\mathrm{mg} / \mathrm{ml}$ in PBS) was added and leave for $2 \mathrm{~h}$. The formed blue color formazan crystal was mixed in $1 \mathrm{ml}$ of DMSO and record the optical density of this solution spectrophotometrically at a particular wavelength $(\lambda=540 \mathrm{~nm})$. Untreated cells with assigned $100 \%$ cell viability were taken as the positive control.

\section{Results and Discussion}

Figure 1a indicates the powder x-ray diffraction (XRD) pattern of as-prepared ZnPc, NORM and ZnPc/NORM. The ZnPc showed sharp diffraction peaks in the XRD spectrum and the peaks match well with the standard monoclinic phase (JCPDS card \#39-1882)
[24]. Similarly, the XRD of NORM and ZnPc/NORM were measured and seen that the diffraction patterns were almost similar, matching the standard spectrum of ZnPc. No peak shift was observed in NORM after ZnPc encapsulation; on the other hand, we observed a decline in the peak's intensity. No ZnPc peaks was observed in $\mathrm{ZnPc} / \mathrm{NORM}$ owing to its encapsulation.

The FTIR spectra for ZnPc, NORM, and ZnPc/NORM are exhibited in figure $1 \mathrm{~b}$. The ZnPc FTIR spectra shows strong peaks around 1112,1056 , and $1083 \mathrm{~cm}^{-1}$ which are characteristic peaks of phthalocyanine ring in accordance with the literature [22]. The NORM FTIR spectrum shows the presence of Si-O-Si at $1110 \mathrm{~cm}^{-1}$ and several other bands due to silanol groups e.g. $1604 \mathrm{~cm}^{-1}(\mathrm{SiO}-\mathrm{H}$ bending), and $1274 \mathrm{~cm}^{-1}$ (Si-0 stretching) bonds and the unhydrolysable amino-groups introduced due to the APTES precursor. For $\mathrm{ZnPc} / \mathrm{NORM}$ spectrum the absorption bands around $2956 \mathrm{~cm}^{-1}$ indicated the presence of $-\mathrm{C}-\mathrm{NH}_{2}$ stretching vibrations which establish amino group incorporation in the synthesized of ZnPc/NORM.
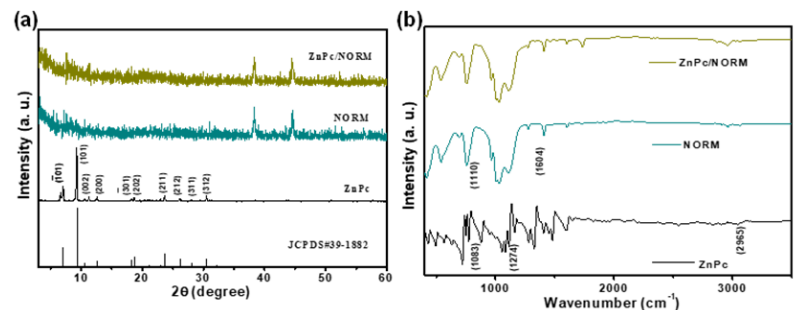

Figure 1: (a) XRD, (b) FTIR spectra of ZnPc, NORM and ZnPc/ NORM.

Furthermore, the NMR spectrum was also taken for $\mathrm{ZnPc} /$ NORM, which is as shown in figure S2 (ESI). In ${ }^{1} \mathrm{H}-\mathrm{NMR}$ all the sixteen hydrogens can be seen. The peak for the eight protons of four rings of the benzene group appeared at $7.55 \mathrm{ppm}$, whereas the rest of eight protons appeared separately at 8.10 and $9.39 \mathrm{ppm}$ for four hydrogens. HRMS spectra [M+H] ${ }^{+}$peak was found at m/z 578.1047 (calculated value: 577.0862) which is in agreement with the molecular formula $\mathrm{C}_{32} \mathrm{H}_{16} \mathrm{~N}_{8} \mathrm{Zn}$ of the compound in figure S3 (ESI). Based on the ${ }^{1} \mathrm{H}-\mathrm{NMR}$ [16] and HRMS spectra the structure of the compound was confirmed. The compound appeared to be blue in color. The absorption spectra of different samples were also taken 
as represent in figure S3 (ESI). The absorption spectrum of NORM does not exhibit any peak while the PS sample show two characteristic peaks centered at 650 and $775 \mathrm{~nm}$. The ZnPc/NORM sample also show same peaks as ZnPc but with less intensity due to the low concentration of ZnPc in NORM/ZnPc.

The morphology of the NORM encapsulated ZnPc particles was observed with EESEM, as shown in figure S1a (ESI). The figure shows the low magnification FESEM image of the sample which confirms the formation of NPs. When a small part of it is magnified, as seen in figure $2 a$, it was seen that the NPs were in spherical shape with size below $100 \mathrm{~nm}$. Furthermore, TEM images of the NPs were taken to study the morphology in precise. Figure $S 2 b$ and figure $2 b$ shows the low and high magnification TEM images of the samples, respectively, which additionally portray that the synthesized particles are spherical in shape with a size range of nearly 50 $\mathrm{nm}$. The high magnification TEM image shows some dark spots inside the spherical particles illustrating the presence of ZnPc inside the NORM, which was also confirmed from the XRD results. In addition, to know the hydrodynamic size distribution of the prepared NPs, DLS spectrum was taken, which shows that the NPs formed were mostly in the size range of $53 \pm 15 \mathrm{~nm}$ (Figure 2c). The result are in well accordance with the TEM images.

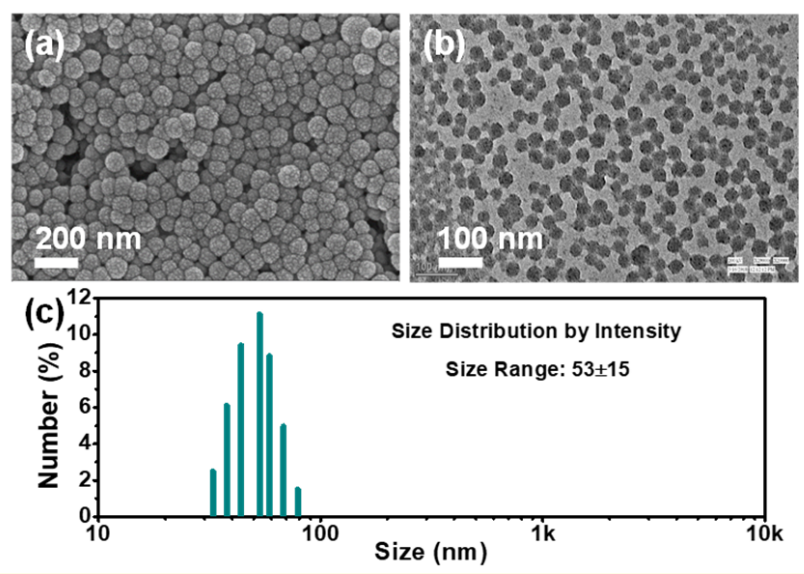

Figure 2: (a) FE-SEM and (b) TEM images, and (c) DLS of ZnPc/NORM.
The porosity of the synthesized ZnPc/NORM NPs was studied using the isothermal adsorption and desorption techniques, as represented in figure 3a. The surface area and porosity of synthesized NPs were studied using BET (nitrogen adsorption isotherm). The specific surface area per unit mass for ZnPc/NORM sample is 177 $\mathrm{m}^{2} / \mathrm{g}$ and Barrett-Joyner-Halenda (BJH) pore diameter of mesoporous silica nanoparticle is $2.17 \mathrm{~nm}$ (inset of figure 3a). Moreover, the BET for synthesized nanoparticle shows type IV mesoporous nature with reference to the IUPAC data and shows a pronounce hysteresis loop for partial pressure (under nitrogen gas) $\mathrm{P} / \mathrm{P}_{0}$ $>0.3$, which confirms the mesoporous structure of the spherical nanoparticle. The mesoporous nanoparticle with high surface area characteristics will have high loading of encapsulated ZnPc (PS) that is favorable to study release kinetics. Time-dependent release of $\mathrm{ZnPc}$ is shown in the figure $3 \mathrm{~b}$. $\mathrm{ZnPc} / \mathrm{NORM}$ was kept in acceptor solution for $80 \mathrm{~h}$ and the release of $\mathrm{ZnPc}$ was observed. The residual content of the PS in the NPs confirms the release data and shows a slow and sustained release at $\mathrm{pH} 7.4$ [39]. There is no fast or burst release behavior which describes the release of PS from ZnPc/NORM NPs is diffusion controlled. (a)

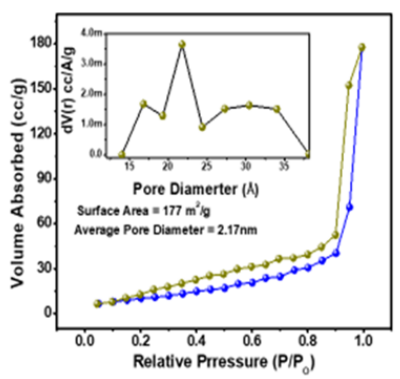

(b)

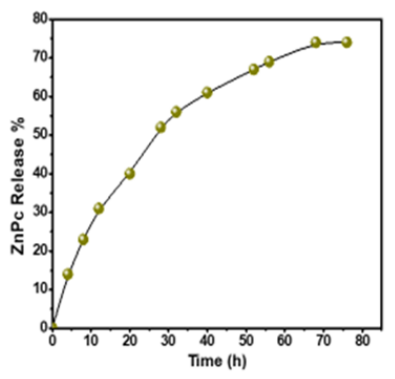

Figure 3: (a) BET (inset shows the pore diameter distribution curve) and (b) Release profile at pH7.4 of ZnPc/NORM.

ABMDMA dye is used as a chemical probe for the photo-induced detection of singlet oxygen $\left({ }^{1} \mathrm{O}_{2}\right)$. The ABMDMA is a water soluble and was photobleached by singlet oxygen $\left({ }^{1} \mathrm{O}_{2}\right)$ to its non-fluorescent derivative endoperoxide. Thus, the formation of endoperoxide clearly display the generation of singlet oxygen $\left({ }^{1} \mathrm{O}_{2}\right)$ and it was 
seen spectrophotometrically by recording the decline in optical density at $400 \mathrm{~nm}$ (corresponding to the absorption $\lambda$ max of ABMDMA), as given in figure 4. First, only ABMDMA dye was irradiated and observed that there is no significant change in absorption with irradiation time (Figure 4a). On the other hand, a slight decrease in absorption was perceived for free $\mathrm{ZnPc}$ and AMBDMA solution (Figure 4b). But when we irradiated ZnPc/NORM and AMBDMA solution, a significant amount of decrease in absorption was observed (Figure 4c). The photobleaching data is summarized in figure $4 \mathrm{~d}$. It can be concluded that significant production of singlet oxygen $\left({ }^{1} \mathrm{O}_{2}\right)$ occurs due to light irradiation on ZnPc/NORM NPs, which thus is a promising platform for PDT [36-39].
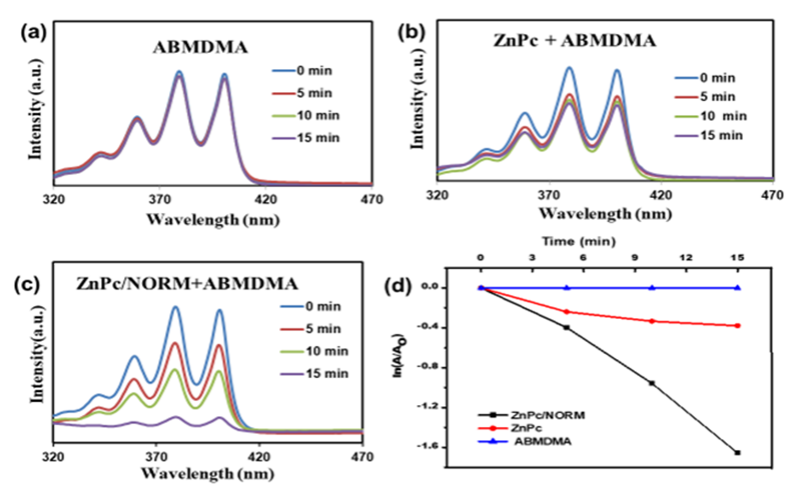

Figure 4: Absorbance versus wavelength plots for the decay of ABMDMA with (a) just ABMDMA, (b) ZnPc with ABMDMA, and (c) ZnPc/NORM with ABMDMA. (d) $\ln (\mathrm{A} / \mathrm{Ao}$ ) versus time plots of all the samples showing the decrease in optical density of ABMDMA at $380 \mathrm{~nm}$.

Photoactivated ROS generation is often accompanied by increase in temperature of the system (photothermal effect) [32]. In order to examine the photothermal effect of ZnPc/NORM, we investigated temperature rise of the system as a part of time of laser light irradiation. Figure 5 shows that comparative temperature rises of aqueous solution of NORM and ZnPc/NORM continuously irradiated with a diode laser light (power density $40 \mathrm{~mW} / \mathrm{cm}^{2}$ ) having wavelength of $635 \mathrm{~nm}$. A pen type digital multi thermometer was utilized to correctly measure the change in temperature of the prepared solution after the light irradiation. From the data it can be observed that a temperature rises of only $0.7^{\circ} \mathrm{C}$ is observed after irradiating NORM sample for a total of 25 minutes. The same exposure conditions on $\mathrm{ZnPc} / \mathrm{NORM}$ led to a temperature rise of $18.9^{\circ} \mathrm{C}$. The result indicates that $\mathrm{ZnPc} / \mathrm{NORM}$ particles also act as good photothermal agents, in addition to their PDT effect. However, the rise in temperature is due to the associated singlet oxygen production or not is unknown at this moment.

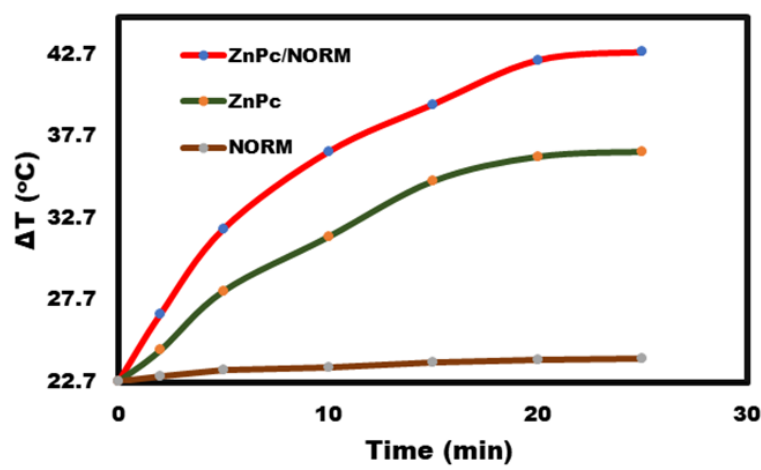

Figure 5: Temperature versus time plots of different samples.

To examine the Cell viability after PDT in vitro, A549 cells were treated with seven different concentration of ZnPc/NORM particles $(20,50,100,150,200,250,300 \mu \mathrm{g} / \mathrm{ml})$ and studied the result by MTT assay $[37,38]$. As shown in figure 6, the ZnPc/NORM particles are non-toxic at all the concentrations tested in the dark (Non-radiated). However, upon light irradiation (Radiated), significant toxicity (about 76\% cell death) is observed at the treatment concentration of $300 \mu \mathrm{g} / \mathrm{ml}$. Cells treated with the pure (free) ZnPc equivalent $(7.8 \mu \mathrm{g} / \mathrm{ml}$ to that of dosage $300 \mu \mathrm{g} / \mathrm{ml}$ for NORM did not show much cytotoxicity (about $21 \%$ cell death) after light exposure. The result are in agreement with the ${ }^{1} \mathrm{O}_{2}$ (singlet oxygen) photogeneration data, where the ZnPc/NORM shows the maximum PDT efficiency.

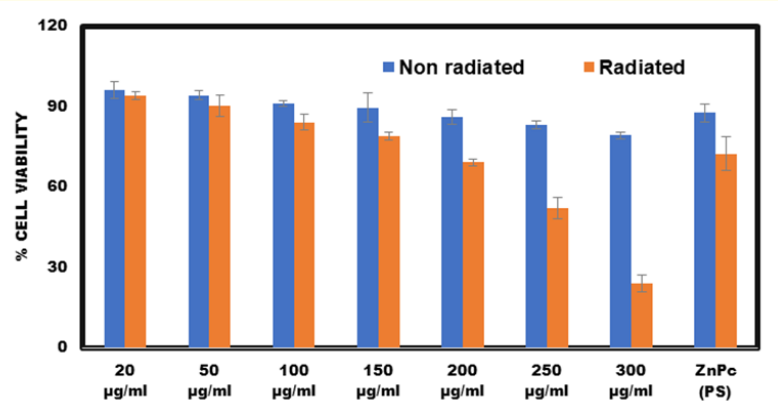

Figure 6: MTT assay studies in A-549 cells treated with ZnPc/ NORM at various concentrations and pure ZnPc, without (Dark) and with (Light) $635 \mathrm{~nm}$ diode laser for $10 \mathrm{~min}$. 

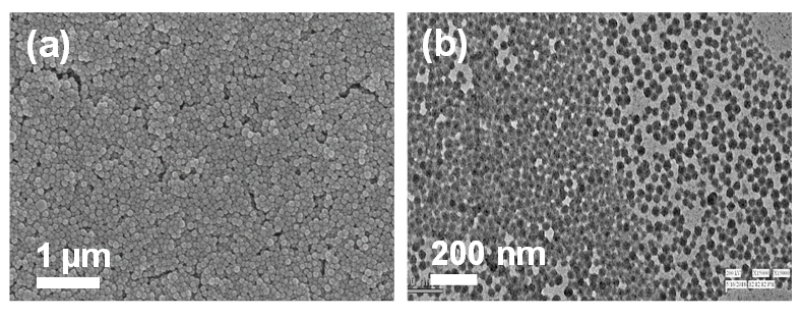

Figure 7: Low magnification (a) FE-SEM and (b) TEM images of $\mathrm{ZnPc} / \mathrm{NORM}$.

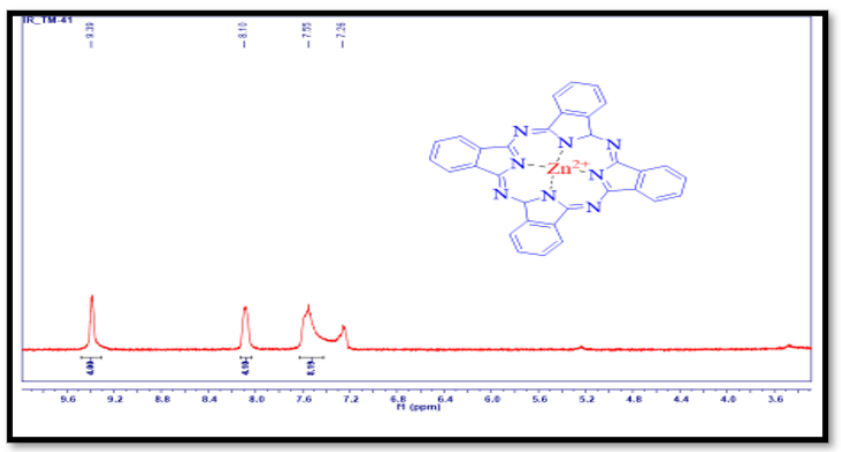

Figure 8: NMR data of Zinc Phthalocyanine (ZnPc).

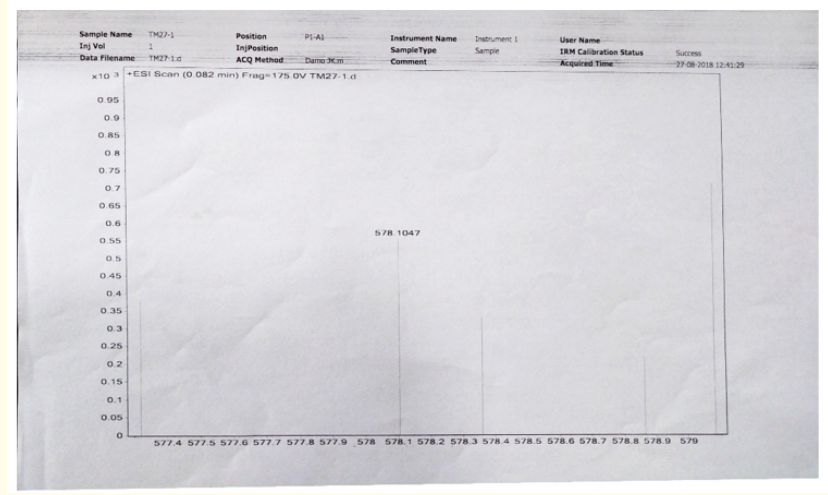

Figure 9: The MALDI-TOF Mass spectra of Zinc phthalocyanine (ZnPc).

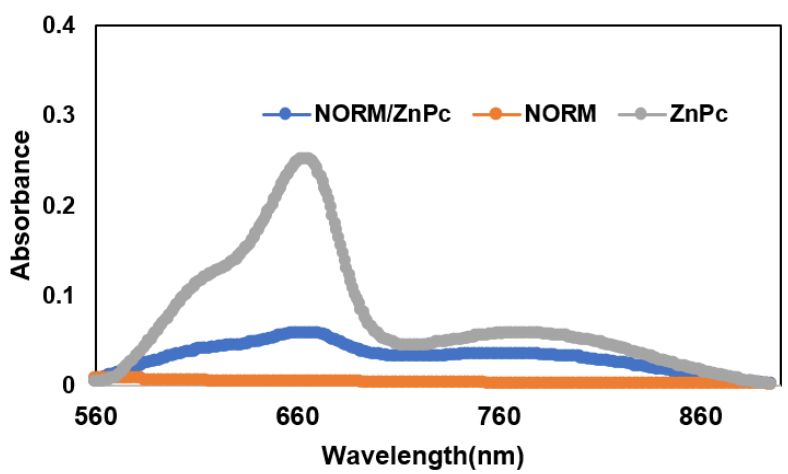

Figure 10: The UV-Visible absorbance Spectra of ZnPc encapsulated NORM.

\section{Conclusion}

In this report, we have synthesized zinc phthalocyanine ( $\mathrm{ZnPc}$ ) nanoparticles embedded in NORM by a simple microemulsion technique. The FESEM and TEM images confirmed the successful encapsulation of ZnPc within the NORM particles with small hydrodynamic size, and good biocompatibility. The XRD and FTIR results of the NORM/PS sample also reinforce the fact that the ZnPc particles are embedded inside the NORM particles. The photobleaching of ABMDMA dye with ZnPc encapsulated NORM shows a greater yield of singlet oxygen species than the pure $\mathrm{ZnPc}$ under laser irradiation. Thus, it can be confirmed that the ZnPc/NORM sample shows good PDT effect than the pure ZnPc. Furthermore, cell viability assays result also showed that the cytotoxicity effect of $\mathrm{ZnPc} / \mathrm{NORM}$ is high and it improves with the enhancement in the concentration of the nanoparticles. However, pure ZnPc showed low cytotoxicity because of less generation of ROS. Encapsulation of ZnPc in the NORM enhanced its stability in an aqueous medium and improved phototoxicity. The ZnPc/NORM sample also showed a good PTT effect with a temperature rise up to $42.8^{\circ} \mathrm{C}$ for $25 \mathrm{~min}$ laser irradiation. The data shows that these $\mathrm{ZnPc} / \mathrm{NORM}$ can be used in sustained drug release applications, and can avoid faster and burst-release behavior witnessed with other drug nanocarriers, including some MOF formulations. 


\section{Supplementary Information}

This manuscript contains Electronic Supplementary Information (ESI), which contain data of 1H-NMR data of Zinc Phthalocyanine (ZnPc), The MALDI-TOF Mass spectra of Zinc phthalocyanine (ZnPc) and the UV-Vis absorbance color spectra of ZnPc encapsulated NORM.

\section{Conflicts of Interest}

There are no confrontations of attention.

\section{Acknowledgements}

TM wants to acknowledge the organization sanction from the CSIR, Government of India. This study was also supported by the UGC-UKIERI (UKIERI-III) research grant. This work was also supported by a grant of the Ministry of Science and Higher Education of the Russian Federation for large scientific projects in priority areas of scientific and technological development (grant number 075-15-2020-774).

\section{Bibliography}

1. Rejeeth C., et al. "Cisplatin-functionalized silica nanoparticles for cancer chemotherapy". Cancer Nanotechnology 4.6 (2013): 127-136.

2. Mi Y., et al. "Application of nanotechnology to cancer radiotherapy". Cancer Nanotechnology 7.1 (2016): 1-16.

3. Singhal S., et al. "Nanotechnology applications in surgical oncology". Annual Review of Medicine 61 (2010): 359-373.

4. Choudhary S., et al. "Photodynamic therapy in dermatology: a review”. Lasers in Medical Science 24.6 (2009): 971-980.

5. Dai T., et al. "Photodynamic therapy for localized infectionsstate of the art". Photodiagnosis and Photodynamic Therapy 6.3-4 (2009): 170-188.

6. Ackroyd R., et al. "The history of photodetection and photodynamic therapy". Photochemistry and Photobiology 74.5 (2001): 656-669.

7. Juarranz Á., et al. "Photodynamic therapy of cancer. Basic principles and applications". Clinical and Translational Oncology 10.3 (2008): 148-154.
8. Castano A P., et al. "Mechanisms in photodynamic therapy: part one-photosensitizers, photochemistry and cellular localization". Photodiagnosis and Photodynamic Therapy 1.4 (2004): 279-293.

9. Wang K., et al. "Self-assembled IR780-loaded transferrin nanoparticles as an imaging, targeting and PDT/PTT agent for cancer therapy". Scientific Reports 6.1 (2016): 1-11.

10. Miura N and Shinohara Y. "Cytotoxic effect and apoptosis induction by silver nanoparticles in HeLa cells". Biochemical and Biophysical Research Communications 390.3 (2009): 733-737.

11. Pan Y., et al. "Gold nanoparticles of diameter $1.4 \mathrm{~nm}$ trigger necrosis by oxidative stress and mitochondrial damage". Small 5.18 (2009): 2067-2076.

12. Fabris C., et al. "Photosensitization with zinc (II) phthalocyanine as a switch in the decision between apoptosis and necrosis". Cancer Research 61.20 (2001): 7495-7500.

13. Tuncel S., et al. "A set of highly water-soluble tetraethyleneglycol-substituted Zn (II) phthalocyanines: synthesis, photochemical and photophysical properties, interaction with plasma proteins and in vitro phototoxicity". Dalton Transactions 40.16 (2011): 4067-4079.

14. Makhseed S., et al. "Water-soluble non-aggregating zinc phthalocyanine and in vitro studies for photodynamic therapy". Chemical Communications 49.95 (2013): 11149-11151.

15. Barbon A., et al. "Photoexcited spin triplet states in zinc phthalocyanine studied by transient EPR". Physical Chemistry Chemical Physics 3.23 (2001): 5342-5347.

16. Anderson C Y., et al. "A comparative analysis of silicon phthalocyanine photosensitizers for in vivo photodynamic therapy of RIF-1 tumors in C3H mice". Photochemistry and Photobiology 67.3 (1998): 332-336.

17. Ping J T., et al. "Synthesis I and optimization of ZnPc-loaded biocompatible nanoparticles for efficient photodynamic therapy". Journal of Materials Chemistry B 4.25 (2019): 4482-4489.

18. Qin J., et al. "Facile synthesis of dual-functional nanoparticles co-loaded with $\mathrm{ZnPc} / \mathrm{Fe} 304$ for PDT and magnetic resonance imaging". Materials Research Bulletin 114 (2019): 90-94. 
19. Wang S., et al. "Synthesis of hemoglobin conjugated polymeric micelle: a ZnPc carrier with oxygen self-compensating ability for photodynamic therapy". Biomacromolecules 16.9 (2015): 2693-2700.

20. Roy I., et al. "Ceramic-based nanoparticles entrapping waterinsoluble photosensitizing anticancer drugs: A novel drugcarrier system for photodynamic therapy". Journal of the American Chemical Society 125.26 (2003): 7860-7865.

21. Samanta M., et al. "Facile synthesis of ZnPc nanoflakes for cold cathode emission". RSC Advances 6.48 (2016): 42739-42744.

22. Wan Y., et al. "Facile in-situ solvothermal method to synthesize ZnPc-MWCNTs composites with enhanced visible light photocatalytic activity". Ceramics International 42 (2): 2425-2430.

23. Gomes A J., et al. "Photobiological and ultrastructural studies of nanoparticles of poly (lactic-co-glycolic acid)-containing bacteriochlorophyll-a as a photosensitizer useful for PDT treatment". Drug Delivery 12.3 (2005): 159-164.

24. Zeisser-Labouèbe M., et al. "Hypericin-loaded nanoparticles for the photodynamic treatment of ovarian cancer". International Journal of Pharmaceutics 326.1-2 (2006): 174-181.

25. Tang W., et al. "Photodynamic Characterization and In Vitro Application of Methylene Blue-containing Nanoparticle Platforms". Photochemistry and Photobiology 81.2 (2005): 242249.

26. Tang W., et al. "Encapsulation of methylene blue in polyacrylamide nanoparticle platforms protects its photodynamic effectiveness". Biochemical and Biophysical Research Communications 369.2 (2008): 579-583.

27. Konan-Kouakou YN., et al. "In vitro and in vivo activities of verteporfin-loaded nanoparticles". Journal of Controlled Release 103.1 (2005): 83-91.

28. Ricci-Júnior E and Marchetti J M. "Preparation, characterization, photocytotoxicity assay of PLGA nanoparticles containing zinc (II) phthalocyanine for photodynamic therapy use". Journal of Microencapsulation 23.5 (2006): 523-538.
29. Chatterjee D K., et al. "Nanoparticles in photodynamic therapy: an emerging paradigm". Advanced Drug Delivery Reviews 60.15 (2008): 1627-1637.

30. Agnihotri S., et al. "Synthesis of Nickel Phthalocyanine Encapsulated ORMOSIL Nanoparticles as Efficient Phototherapeutic Agent". Advanced Science, Engineering and Medicine 10.1 (2018): 22-26.

31. Yurt F., et al. "Investigation of in vitro PDT activities of zinc phthalocyanine immobilised TiO2 nanoparticles". International Journal of Pharmaceutics 524.1-2 (2017): 467-474.

32. Yang T., et al. "Ultrastable near-infrared conjugated-polymer nanoparticles for dually photoactive tumor inhibition". Advanced Materials 29.31 (2017): 1700487.

33. Roy I. "Gold Nanoparticle-Enhanced Photodynamic Therapy from Photosensitiser-Entrapped Ormosil Nanoparticles". Journal of Nanoscience and Nanotechnology 19.11 (2019): 69426948.

34. Muehlmann L A., et al. "Aluminium-phthalocyanine chloride nanoemulsions for anticancer photodynamic therapy: Development and in vitro activity against monolayers and spheroids of human mammary adenocarcinoma MCF-7 cells". Journal of Nanobiotechnology 13.1 (2015): 1-11.

35. Song M R., et al. "Zeolitic imidazolate metal organic framework-8 as an efficient $\mathrm{pH}$-controlled delivery vehicle for zinc phthalocyanine in photodynamic therapy". Journal of Materials Science 534 (2018): 2351-2361.

36. Peng J., et al. "Hollow silica nanoparticles loaded with hydrophobic phthalocyanine for near-infrared photodynamic and photothermal combination therapy". Biomaterials 34.32 (2013): 7905-7912.

37. Sethi K and Roy I. "Organically modified titania nanoparticles for sustained drug release applications". Journal of Colloid and Interface Science 456 (2015): 59-65.

38. Jain S K., et al. "Calcium silicate-based microspheres of repaglinide for gastroretentive floating drug delivery: Preparation and in vitro characterization". Journal of Controlled Release 107.2 (2005): 300-309. 
39. Sharma S., et al. "Magnetic nanoscale metal-organic frameworks for magnetically aided drug delivery and photodynamic therapy". New Journal of Chemistry 41.20 (2017): 1186011866.

Volume 5 Issue 7 July 2021

(C) All rights are reserved by Tarun Mohan., et al. 